\title{
A NOVEL CROSS-LAYER DESIGN FOR DYNAMIC TXOP IN IEEE802.11e
}

\author{
Jun Tu, Dehua Li, Xuesi Liu
}

Original scientific paper This paper proposes a novel framework for the cross-layer design and optimization of wireless networks combining adaptive modulation and coding (AMC) at the physical (PHY) layer by means of automatic repeat request and channel-aware multiuser scheduling protocols at the data link control (DLC) layer. The proposed framework is based on the use of two-dimensional discrete time Markov chains (DTMCs) jointly modelling the AMC scheme and the amplitude contention window (CW). A new algorithm for dynamic configuration of the polled transmission opportunity (TXOP) in multi-rate IEEE $802.11 \mathrm{e}$ network is presented and its performance is analysed and compared with default configuration. The numerical simulation results have indicated the high efficacy of the proposed method in maximizing the average throughput of the system.

Keywords: Adaptive Modulation and Coding (AMC); cross-layer design; Hybrid Automatic Repeat Request (HARQ); Markov models

\section{Novi cross-layer projekt za dinamički TXOP u IEEE802.11e}

Izvorni znanstveni članak

U radu se predlaže novi okvir za konstrukciju s poprečnim slojevima (cross-layer design) i optimizaciju bežičnih mreža kombiniranjem adaptivne modulacije i kodiranja (AMC) na fizičkom sloju (PHY) pomoću automatskog zahtjeva za ponavljanjem (automatic repeat request) i channel-aware multiuser scheduling protokola kod sloja za reguliranje povezivanja podataka - data link control (DLC). Predloženo se načelo zasniva na primjeni dvodimenzijskih Markovljevih lanaca diskretnog vremena - two-dimensional discrete time Markov chains (DTMCs) koji skupa modeliraju AMC shemu i amplitude contention window (CW). Predstavljen je novi algoritam za dinamičku konfiguraciju ispitivane mogućnosti prijenosa - polled transmission opportunity (TXOP) u IEEE 802.11e mreži s više brzina te se analizira njegova učinkovitost $\mathrm{i}$ uspoređuje sa zadanom konfiguracijom. Rezultati numeričke simulacije pokazali su visoku učinkovitost predložene metode u maksimiranju prosječnog protoka sustava.

Ključne riječi: adaptivna modulacija i kodiranje (AMC); cross-layer konstrukcija; hibridni automatski zahtjev za ponavljanjem(HARQ); Markovljevi modeli

\section{Introduction}

Scheduling and hybrid automatic repeat request (HARQ) error control protocols at the media access control (MAC) sub-layer and adaptive modulation and coding (AMC) strategies at the physical (PHY) layer are some of the key technologies underpinning state-of-theart and next-generation wireless communication systems [1]. They are used to optimize resource utilization while providing support to a wide range of multimedia applications with heterogeneous quality of service (QoS) requirements [2].

In IEEE 802.11e, accessing to the shared wireless channel is controlled through two MAC layer mechanisms $[3 \div 5]$, i.e. polling based point coordination function (PCF) and contention based distributed coordination function (DCF).

The 802.11e enhances the DCF and the PCF by a new coordination function, i.e. the hybrid coordination function (HCF). Within the HCF, there are two methods to access channel, i.e. HCF controlled channel access (HCCA) and enhanced distributed channel access (EDCA) $[4 \div 6]$. The HCCA mechanism, the so called polled transmission opportunity (TXOP), is that when a station is polled, it is allowed to transmit packets holding the channel up to a maximum time interval [7]. The 802.11e allows the definition of a contention period for the EDCA and a contention free period for HCCA [8]. In this way, performance enhancement is achieved by using the transmission on single channel access. A station is allowed to send a number of consecutive packets limited by the duration of allocated TXOP. Therefore, the lack of an efficient configuration of the TXOP duration may result in wasteful bandwidth sharing a slot time, resulting in delay, jitter and throughput reduction of the system. To solve this issue the dynamic cross-layer design method has been proposed to enhance the accuracy of TXOP duration estimation [9].

Literature review indicates that much work has focused on cross-layer designs by combining the AMC schemes with HARQ or ARQ error control protocols [911] while limited researches have addressed the multiuser scheduling at the MAC sub-layer [12 $\div 15]$. In [15] the authors presented an opportunistic scheduling scheme to improve the delay performance of secondary users with bursty traffic in cognitive radio (CR) systems. They considered a relay-assisted CR network with a decodeand-forward relaying scheme. Cooperative beam forming was used by the relays to forward packets in either idle or busy time slots without causing interference to primary users. However, in the research, although there was a scheduler to plan the transmissions of the source and the relays, only one user was considered and the use of AMC was not taken into account. In [14] the authors presented the multiuser scheduling process based on the first-order amplitude-based finite state Markov chain (AFSMC) model. The max-rate multiuser scheduler was included in their model through a service-vacation process. This model allowed a manageable number of system states irrespective of the number of users sharing the channel. However, the model in [14] did not define a crosslayering scheme to optimize the system performance. As a result, the users were assumed to operate in channels with equal characteristics, restricting the usefulness of the presented results. In [13] the authors developed a theoretical framework based on a finite-state Markov chain (FSMC) to model a heterogeneous multiuser scenario where groups of users coexisted with different 
QoS requirements. In this analysis, it was assumed that the Markov chain steady-state probabilities of any user could be considered independent from the steady-state probabilities of all the other users in the system. Furthermore, it was assumed that the steady-state probabilities of different users belonging to the same QoS class were identical. These assumptions restricted the possible application scenarios of the FSMC because they implied that the traffic and channel characteristics were exactly the same for all users belonging to the same QoS class. This problem could be solved by proper cross-layer design; however, little related work was reported in this research filed.

In order to address the above issue, this paper proposes a novel framework for the cross-layer design and optimization of wireless networks by combining $\mathrm{AMC}$ at the PHY layer. The two-dimensional discrete time Markov chains (DTMCs) have been employed to jointly model the AMC and the amplitude contention window (CW) to obtain the suitable cross-layer design. Numerical analysis results show the effectiveness of the proposed method in the cross-layer design for Dynamic TXOP in IEEE802.11e.

\section{A Cross-Layer Design model for TXOP scheduler}

TXOP can be obtained by two ways of competition or distribution, which are called EDCA TXOP and HCCA TXOP, respectively [16]. TXOP mechanism and the priority mechanism of EDCA can effectively guarantee high-priority business of QoS and improve the performance of high-priority business. However, for all sites, it is not fair to use the same fixed value of the TXOP limit [17]. If the TXOP limit is a fixed value, it is easy to decrease the performance of low priority business, causing the situation of "starvation". The essence of EDCA access mechanism is set by the channel access parameters, making the data get QoS in a certain advantage over its competition. This advantage is obtained by "damaging" the "interests" of some lowpriority data. Because the bandwidth is certain, in order to ensure the throughput and delay of high-priority data, it is necessary to decrease and increase the throughput and delay of low-priority data, respectively.

Under the HCCA mode, the duration of TXOP is not a simply fixed value, but determined by a simple scheduling mechanism. After the hybrid coordinator (HC) receiving the request, $\mathrm{HC}$ calculates whether each $\mathrm{TS}$ can transmit frame or not, according to the parameters of the request. The algorithm of HCCA TXOP is based on the polling mechanism. If the state information (SI) is too small, it makes TXOP unnecessary with the frequent allocation of $\mathrm{HC}$, adding to the polling burden of $\mathrm{HC}$. Thus, we design a cross-layer model based on AMC, as illustrated in Fig. 1.

At the base station (BS), there are $N s$ separate radio link level buffers that are used to queue packet arrivals corresponding to every user connected to the BS. These buffers operate in a first-in-first-out (FIFO) manner and can store up to $\boldsymbol{Q}=\left\{Q_{1}, Q_{2} \ldots, Q_{u} \ldots, Q_{N_{s}}\right\}$ packets, where $Q_{u}$ is the queue length of user $u$. Adaptive transmission is performed by using an HARQ error control scheme at the MAC layer and an AMC strategy at the PHY layer. The processing unit at the MAC layer is a packet and the processing unit at the PHY layer is a frame. We propose a new scheduling mechanism, which can dynamically adjust the SI of quality of service aware station (QSTA) to schedule the data stream of sites and make the scheduling scheme more efficient. AMC of information transmit cross-layer controller by channel state information (CSI). The link is assumed to support QoS-guaranteed traffic characterized by a maximum average packet delay and a target link layer packet loss rate (PLR) to adapt to the transmission of media streaming and the improvement of wireless network throughput, average data transmission delay, packet loss rate and so on [18].

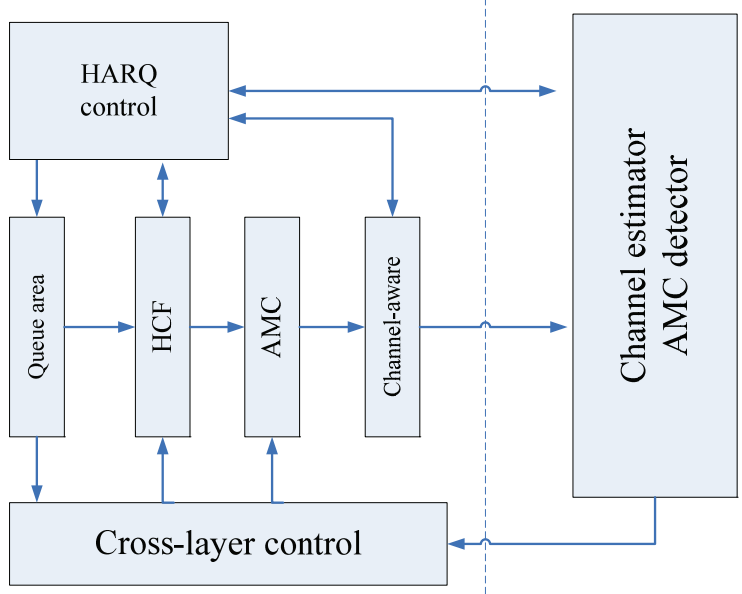

Figure 1System model

\section{Markov model \& dynamic TXOP scheduler}

The AMC scheme is assumed to have a set of $M$ possible transmission modes (TMs) $(\boldsymbol{M}=\{0 \ldots, M-1\})$. Each TM corresponds to a particular combination of modulation and coding strategies.

It is assumed that when the system uses $\mathrm{TM} n \in \mathrm{M} p$, it transmits $p n(p n=b \mathrm{R} n)$ packets per frame, where $\mathrm{R} n$ denotes the number of information bits per symbol used by $\mathrm{TM} n$ and $b$ is a parameter that determines the number of transmitted packets per frame. As shown in [9], depending on the channel conditions and the QoS requirements of different users, some of these possible TMs may be deemed useless, and thus, only a set of $M u$ useful TMs $(\boldsymbol{M}=\{0 \ldots, M u-1\})$ will be available to the AMC scheme for user $u$.

\subsection{Adaptive modulation and coding}

Let denote the instantaneous received signal-to-noise ratio (SNR) of user $u$ using a time frame interval. It is assumed that the frame duration is smaller than the coherence time of the channel and allowed to vary across successive frame intervals. For the assumed Rayleigh block-fading channel model [19], the frame duration can be modelled as an exponentially distributed random variable with mean and standard deviation. Given that the objective of AMC aims to select the TM with maximum data rate, the average packet error ratio (PER) is required to be less than or equal to a prescribed value. To this end, according to [3], the entire SNR range is partitioned into a 
set of non-over lapping intervals defined by the partition to determine TM $n$. In this paper, the partition is obtained by using the threshold searching algorithm described in [10]. This searching algorithm has the capability to discriminate between useful and useless TMs and at the same time to guaranty the required average PER.

\subsection{Joint PHY-MAC layer Markov model}

Channel-aware-only schedulers can be incorporated into the joint PHY-MAC Markov model by means of service-vacation process [14]. When a particular user $u$ is selected for transmission in a given time slot, the PHY layer of this user is in service; otherwise, it is on vacation. The decision whether the user $u$ is in service or vacation during the next time slot will depend on the possible PHY layer states of all users in the next time slot and their previous scheduling decisions.

The joint PHY-MAC layer FSMC state for user $u$ is denoted by the vector of random variables. At any time, the joint PHY-MAC layer state can be univocally identified by an integer number. Taking into account that user $u$ transmits only when it is in service, the different PHY-MAC states will have a transmission rate (TR) to measure the packets per slot in PHY layer. Furthermore, the PHY-MAC layer FSMC will be described by a transition probability matrix with state transition probabilities that can be analytically calculated for a significant number of scheduling schemes.

$P_{i, j}^{u}=\operatorname{Pr}\left\{n_{v+1}^{u}=j \mid n_{v}^{u}=i\right\}$,

where, $P_{i, j}^{u}$ is the transition probability of user $u ; \operatorname{Pr}$ is the state transition probabilities.

In one-step memory service-vacation processes, scheduling decisions only rely on the actual system state, and thus, the state transition probabilities in Eq. (1) can be simplified. The transition probability matrix can be expressed as

$P_{q}^{u}=\left(\begin{array}{ll}q_{0,0}^{u} & q_{0,1}^{u} \\ q_{1,0}^{u} & q_{1,1}^{u}\end{array}\right)$

where $q_{i, j}^{u}$ is a matrix element. Without loss of generality, user $u(u=1)$ is considered as the user of interest. Taking into account that the PHY layer and service-vacation processes are independent, the elements of the state transition probability matrix can be written as

$$
\begin{aligned}
& \operatorname{Pr}\left\{z_{v+1}, y_{v+1} \mid z_{v}, y_{v}\right\}= \\
& \operatorname{Pr}\left\{z_{v+1} \mid z_{v}, y_{v+1}, y_{v}\right\} \operatorname{Pr}\left\{y_{v+1} \mid y_{v}\right\}
\end{aligned}
$$

The latter term is an element of the PHY layer state transition probability matrix [20]. In order to obtain $\mathrm{Pr}$, we discuss the following two different conditions.

(1) Calculation of $\operatorname{Pr}\left\{z_{v}=0 \mid y_{v}=k\right\}$

Assuming $z$ the denominator of Eq. (3) can be calculated as:

$$
\begin{aligned}
& \operatorname{Pr}\left\{z_{v}=0 \mid y_{v}=k\right\}=\sum_{y_{v}^{2}=0}^{\widehat{N}_{P H Y}^{2}} \sum_{y_{v}^{3}=0}^{\widehat{N}_{P H Y}^{3}} \ldots \sum_{y_{v}^{N_{S}}=0}^{\widehat{N}_{S}^{N_{S}}} \operatorname{Pr}\left\{z_{v}=\right. \\
& \left.0, y_{v}^{2}, y_{v}^{3}, \ldots, y_{v}^{N_{S}} \mid y_{v}=k\right\} .
\end{aligned}
$$

where, $\widehat{N}_{P H Y}^{u} \triangleq 2 N_{P H Y}^{u}-1$. At time slot $v$, the PHY layer of user $u$ is in state $k$ and can only be in service if the rest of users have a PHY layer state with a lower or equal TR. When a user can transmit at maximum $\mathrm{TR}$, user $u$ is chosen for transmission with a probability $1 / a$.

$$
\begin{aligned}
& \operatorname{Pr}\left\{z_{v}=0, y_{v}^{2}, y_{v}^{3}, \ldots, y_{v}^{N_{s}} \mid y_{v}=k\right\}= \\
& 0, \exists u \in U: c_{y_{v}^{u}}^{u} c_{k} \\
& \left\{\begin{array}{c}
1 \\
\frac{1}{a} \prod_{u=2}^{N_{s}} P_{P H Y}^{u}\left(y_{v}^{u}\right), \exists\left\{u_{i} \in U\right\}_{i=1}^{a-1}: c_{y_{v}} u_{i}=c_{k} \forall i
\end{array}\right.
\end{aligned}
$$

where $U=\left\{2, \ldots, N_{s}\right\}$ is the set of competitor users and $c_{k}$ is the TR corresponding to $y_{v}=k$.

(2) Calculation of $\operatorname{Pr}\left\{z_{v+1}, z_{v} \mid y_{v+1}, y_{v}\right\}$

The numerator of Eq. (3) can be written as

$\operatorname{Pr}\left\{z_{v}=z, z_{v+1}=v \mid y_{v}=k, y_{v+1}=1\right\}=$ $\sum_{y_{v+1}^{2}=0}^{\widehat{N}_{P H Y}^{2}} \ldots \sum_{y_{v+1}^{N_{S}}=0}^{\widehat{N}_{S}^{N_{S}}} \sum_{y_{v}^{2}}^{\widehat{N}_{P H Y}^{2}} \ldots \sum_{y_{v}^{N_{S}=0}}^{\widehat{N}_{P H}^{N_{S}}} \operatorname{Pr}\left\{z_{v}=z, z_{v+1}=\right.$

$\left.v, y_{v}^{2}, \ldots, y_{v}^{N_{S}}, y_{v+1}^{2}, \ldots, y_{v+1}^{N_{S}} \mid y_{v}=k, y_{v+1}=1\right\}$.

This paper has proposed a proportional fair (PF) scheduler promises a trade-off between the maximization of average throughput and system fairness. At each time slot, the user experiencing the highest instantaneous rate with respect to its average rate is scheduled. That is, user $q$ is selected for transmission during time slot $v$ if

$q=\arg \max _{u \in\left\{1 \ldots, N_{S}\right\}} \frac{C_{y_{v}^{u}}}{T_{v}^{u}}$.

The average rate can be computed as a moving average over a time window of length $W$.

$T_{v+1}^{u}=\left(1-\frac{1}{W}\right) T_{v}^{u}+\left(1-z_{v}^{u}\right) \frac{1}{W} c_{y_{v}} u$

Through the integration of $W$ we can find TOXP-limit value.

$W=\frac{T_{v}^{u}+C_{y_{v} u-Z_{v}^{u}}^{u}}{T_{v}^{u}-T_{v+1}^{u}}$

\section{TOXP dynamic adjustment algorithm}

Each site obtains the authority to access the channel and transmit its data in the TXOP time defined by HCCA of IEEE802. 11e. In this case, if we use the control method mentioned above, it will generate unnecessary delays which causes the loss of transmission opportunities. To avoid this problem, the proposed algorithm adaptively adjusts the bias deviation between the actual time and the expected time. Therefore, the twice allocation algorithm can flexibly control the TXOP duration of data transmission and improve the transmission efficiency at each site.

$T D_{i}=\operatorname{Max}\left(\frac{u s_{i} \times \rho_{i}}{R_{i}}+O, \frac{M_{i}}{R_{i}}+O\right)$.

where, $T D_{i}$ denotes the duration time of QSTAi's TXOP, $R i$ denotes the minimum physical bit rate, $M$ denotes the maximum MSDU, and $O$ denotes extra cost. 
Finally the access control scheduler is employed to determine the new TS.

$\frac{T D_{k+1}}{S_{i}}+\sum_{i=1}^{k} \frac{T D_{i}}{S_{i}} \leq \frac{T-T c p}{T}$.

where, $k$ denotes the current quantity of TS, $k+1$ denotes the new arrival TS, $T$ denotes the interval of beacon, $T c p$ denotes the transmission time of EDCA business.

\section{Experiments and results analysis}

In order to improve the QoS performance of audio data transmission in wireless local area network (WLAN), the numerical simulation is carried out to examine the performance of the proposed HCCA algorithm. In the simulation, the average delay of data transmission, packet loss rate, throughput and TXOP transmission efficiency are chosen as the metrics to evaluate QoS.

Additionally, some adaptation referring to SI, considered in our algorithm, was introduced. Explicitly, the TXOP limit parameter is computed by the minimum SI, which is permitted by IEEE 802.11 MAC sub-layer and PHY layer for discrete time Markov chains (DTMCs). This assumption normalizes the duration of TXOP intervals when nodes are beyond the range of the fastest PHY layer.

As for the throughput, it can be calculated using the amount of bytes; the transmission delay is defined as the time from sending the source to receiving the frame; the packet loss rate is defined as the ratio of the number of lost packets and the total number of packets.

In our simulation, G711 Voice signals have been used and the parameters are listed in Tab. 1.

Table 1 Parameters specification

\begin{tabular}{ll}
\hline Mean Data rate (Kbps) & 80 \\
\hline Delay bound (bytes) & 60 \\
Nominal MSDU size (bytes) & 210 \\
Maximum delay (ms) & 40 \\
Maximum burst size (bytes) & 578 \\
Peak data rate (Kbps) & 83 \\
Minimum PHY Rate (Mbps) & 12 \\
Minimum service interval (ms) & 20 \\
Maximum service interval (ms) & 30 \\
\hline
\end{tabular}

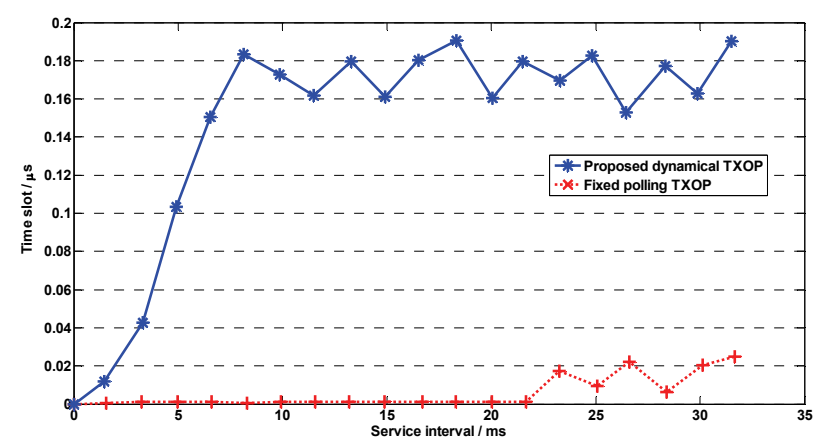

Figure 2 Average data transmission delay

The simulation has compared the fixed polling TXOP with dynamic TXOP. Figs. $2 \div 5$ show the comparison result. Because of the extra cost of computation on a variety of load data flow, it can be seen in Fig. 2 that IEEE802. 11e simple scheduling algorithm (Simple
Scheduler) achieves a low average delay. However, the delay of the proposed dynamic algorithm meets the requirements of QoS. Hence, the performance of the proposed method is higher than the Simple Scheduler with respect to the average data transmission delay.

Fig. 3 shows the packet loss rate results. One can note in Fig. 3 that when the number of sites is more than five, the packet loss rate of the proposed dynamic algorithm is between $0,8 \%$ and $1,5 \%$ while the packet loss rate of Simple Scheduler increases rapidly. When the number of sites is 11 , the packet loss rate of the proposed dynamic algorithm stabilizes around $50 \%$. Hence, the performance of the proposed method is better than the Simple Scheduler with respect to the packet loss rate.

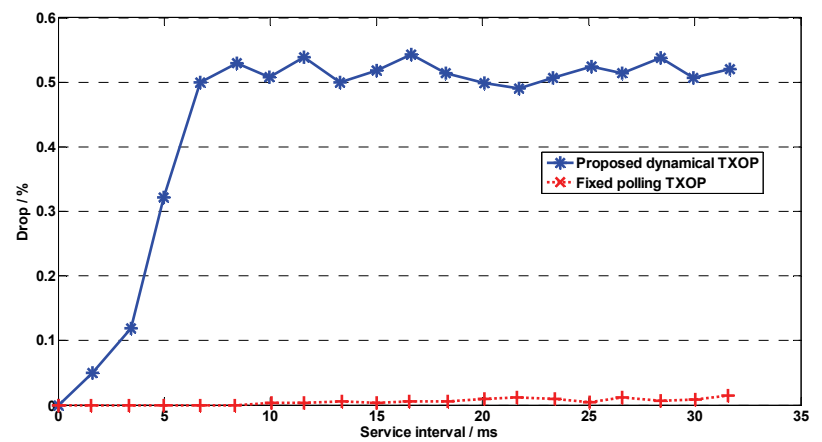

Figure 3 Packet loss rate

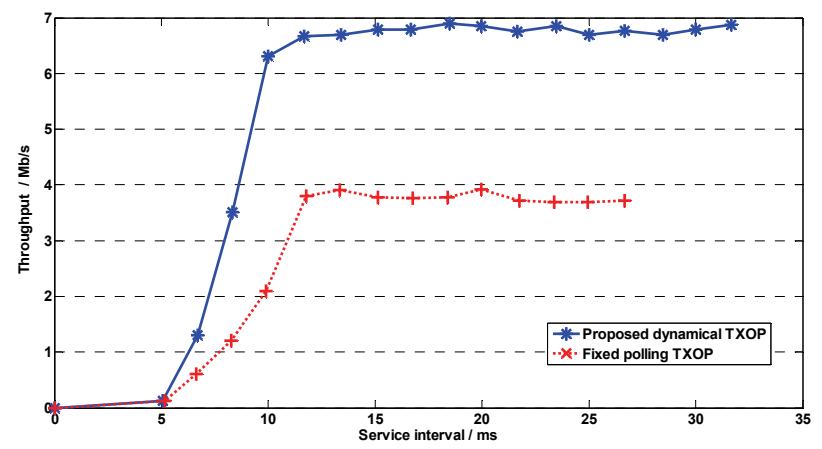

Figure 4 Throughput

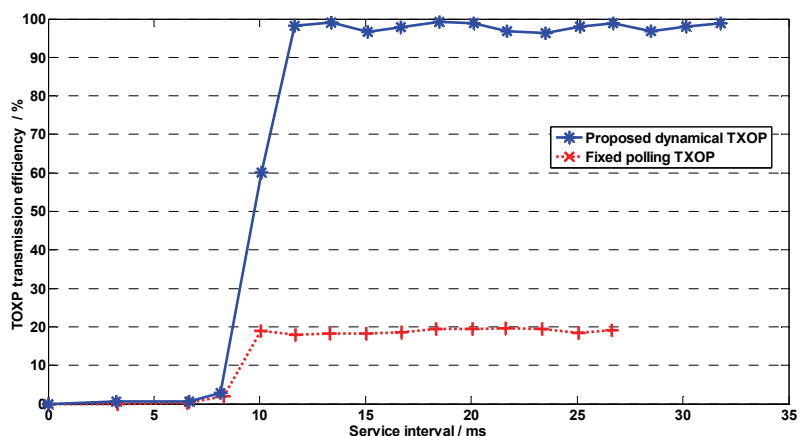

Figure 5 TOXP transmission efficiency

Fig. 4 shows the throughput results. When the number of sites is more than five, owing to the fixed TXOP duration, the increase of throughput in the Simple Scheduler is not remarkable and the stable value is about $4 \mathrm{Mb} / \mathrm{s}$. However, the throughput of the proposed method increases up to near $7 \mathrm{Mb} / \mathrm{s}$. The improvement on throughput and loss rate of the proposed dynamic algorithm mainly benefits from the accurate allocation of transmission opportunities. Hence, the performance of the 
proposed method is higher than the Simple Scheduler with respect to the average data transmission delay.

Fig. 5 shows the TOXP transmission efficiency results. Because the TXOP is calculated according to requirements, the utilization ratio achieves $90 \%$ by the proposed dynamical TXOP while the fixed TXOP scores $20 \%$. As a result, the proposed algorithm has improved the QoS performance of the system.

\section{Conclusion}

This paper has proposed a new scheduler in IEEE802.11e using dynamical TXOP, which collects the information of Ns users based on channel state information (CSI). This new method provides a dynamic time division for multiplexing scheme and takes scheduling decisions to allocate transmission opportunities to active users. Owing to the strong dependencies between MAC and PHY layers in wireless networks, the system efficacy may not be warranted by using a strictly layered optimization approach. Consequently, the proposed new dynamical cross-layer design is able to jointly optimize the scheduling, HARQ, and $\mathrm{AMC}$.

The contribution of this paper is that the HCCA scheduler is applied for distributing network resources dynamically according to each site's traffic stream. By doing so, the TXOP duration time of traffic transfer in the second distribution scheduler could be managed flexibly, and thus improves the transfer efficiency on each site. The numerical simulation analysis demonstrates that the proposed dynamical cross-layer design method in IEEE802.11ecould not only improve the efficiency of the WLAN, but also meets the need of real time business QoS. Thus, the newly proposed dynamical method has practical application importance.

\section{References}

[1] Liu, Q.; Zhou, S.; Giannakis, G. Cross-layer combining of adaptive modulation and coding with truncated ARQ over wireless links. // IEEE Trans. Wireless Commun. 3, 5(2004), pp. 1746-1755.DOI: 10.1109/TWC.2004.833474

[2] Liu, Q.; Zhou, S.; Giannakis, G. Queuing with adaptive modulation and coding over wireless links: cross-layer analysis and design. // IEEE Trans. Wireless Commun. 4, 3(2005), pp. 1142-1153.DOI: 10.1109/TWC.2005.847005

[3] Liu, Q.; Zhou, S.; Giannakis, G. Cross-layer scheduling with prescribed QoS guarantees in adaptive wireless networks. // IEEE J. Selected Areas Commun. 23, 5(2005), pp. 1056-1066.DOI: 10.1109/JSAC.2005.845430

[4] Le, L.; Hossain, E.; Alfa, A. Service differentiation in multirate wireless networks with weighted round-robin scheduling and ARQ-based error control. // IEEE Trans. Commun. 54, 2(2006), pp. 208-215. DOI: 10.1109/TCOMM.2005.863788

[5] Le, L.; Hossain, E.; Alfa, A. Radio link level performance evaluation in wireless networks using multi-rate transmission with ARQ-based error control. // IEEE Trans. Wireless Commun. 5, 10(2006), pp. 2647-2653. DOl: 10.1109/TWC.2006.04485

[6] Ishizaki, F.; Hwang, G. Cross-layer design and analysis of wireless networks using the effectivebandwidth function. // IEEE Trans. Wireless Commun. 6, 9(2007), pp. 3214-3219. DOI: 10.1109/TWC.2007.06030108
[7] Poggioni, M.; Rugini, L.; Banelli, P. Analyzing Performance of Multi-User Scheduling Jointly with AMC and ARQ. // Proceedings of the IEEE Global Telecommunications Conference (GLOBECOM) / Washington D.C., 2007, pp. 3483-3488. DOI: 10.1109/glocom.2007.661

[8] Wang, X.; Liu, Q.; Giannakis, G. Analyzing and optimizing adaptive modulation coding jointly with ARQ for QoSguaranteed traffic. // IEEE Trans.Veh. Technol. 56, 2(2007), pp. 710-720. DOI: 10.1109/TVT.2007.891465

[9] Ramis, J.; Carrasco, L.; Femenias, G. A Two-Dimensional Markov Model for Cross-Layer Design in AMC/ARQbased Wireless Networks. // Proceedings of the IEEE GLOBECOM / New Orleans, 2008, pp. 4637-4642. DOI: 10.1109/glocom.2008.ecp.888

[10] Femenias, G.; Carrasco, L.; Ramis, J. Using twodimensional Markov models and the effective-capacity approach for cross-layer design in AMC/ARQ-based wireless networks. // IEEE Trans. Veh. Technol. 58, 8 (2009), pp. 4193-4203. DOI: 10.1109/TVT.2009.2020723

[11] Ramis, J.; Femenias, G.; Riera, F.; Carrasco, L. CrossLayer Optimization of Adaptive Multirate Wireless Networks using Truncated Chase Combining HARQ. // Proceedings of the IEEE GLOBECOM / Miami, 2010, pp. 6-10.

[12] Ramis, J.; Femenias, G. Cross-layer design of adaptive multirate wireless networks using truncated HARQ. // IEEE Trans. Veh. Technol. 60, 3(2011), pp. 944-954. DOI: 10.1109/TVT.2011.2108324

[13] Poggioni, M.; Rugini, L.; Banelli, P. QoS analysis of a scheduling policy for heterogeneous users employing AMC jointly with ARQ. // IEEE Trans. Commun. 58(2010), pp. 9-23. DOI: 10.1109/TCOMM.2010.09.090276

[14] Le, L.; Hossain, E.; Alfa, A. Delay statistics and throughput performance for multi-rate wireless networks under multiuser diversity. // IEEE Trans. Wireless Commun. 5, 11(2006), pp. 3234-3243. DOI: 10.1109/TWC.2006.04880

[15] Liu, J.; Chen, W.; Cao, Z.; Zhang, Y. Delay optimal scheduling for cognitive radios with cooperative beamforming: a structured matrix-geometric method. // IEEE Trans. Mobile Comput. 11, 8(2012), pp.1412-1423. DOI: 10.1109/TMC.2011.153

[16] Yamane, T. An Efficient Assignment of Transmission Opportunity in QoS Guaranteed Wireless LAN. // Seventh International Conference on Parallel and Distributed Computing, Applications and Technologies, 2006. PDCAT '06. / Taipei, 2006, pp.105-108. DOI: 10.1109/pdcat.2006.30

[17] Alessandro, A.; Riccardo, Z. QoS Enhancement with Dynamic TXOP Allocation in IEEE 802.11e. // The 18th Annual IEEE International Symposium on Personal, Indoor and Mobile Radio Communications / Athens, 2008, pp.245257.

[18] Wu, D.; Negi, R. Effective capacity: a wireless link model for support of quality of service. // IEEE Trans. Wireless Commun. 2, 4(2003), pp.630-643.

[19] Biglieri, E.; Caire, G.; Taricco, G. Limiting performance of block fading channels with multiple antennas. // IEEE Trans. Inf. Theory 47, 4(2001), pp.1273-1289. DOI: 10.1109/18.923715

[20] Carrasco, L.; Femenias, G.; Ramis, J. Channel-aware MAC performance of AMC-ARQ-based wireless systems. // EURASIP Journal on Wireless Communications and Networking, (2013), pp.356-377. DOI: 10.1186/1687-14992013-213 


\section{Authors' addresses}

\section{A. Prof. Dr Jun Tu}

1) School of Automation, Huazhong University of Science

Technology, Wuhan, China

2) School of Computer Science and Technology, Hubei University

of Technology, Wuhan, China

\section{Prof. Dr Dehua Li}

School of Automation, Huazhong University of Science

Technology, Wuhan, China

E-mail: lidh_hust@163.com

\section{Mrs Xuesi Liu}

School of Foreign Languages, Hubei University of Technology,

Wuhan, China 